\title{
Physiological Responses During the Lower Body Positive Pressure Supported Treadmill Test
}

\author{
Ka-Young Lee, $\mathrm{MD}^{1,2}$, Jae-Young Han, MD, PhD ${ }^{1,2}$, Ji-Hyun Kim, $\mathrm{MD}^{1,2}$, \\ Dong-Ju Kim, $\mathrm{MD}^{1,2}$, In-Sung Choi, $\mathrm{MD}, \mathrm{PhD}^{1,2}$
}

${ }^{1}$ Department of Physical and Rehabilitation Medicine, Regional Cardiocerebrovascular Center, Chonnam National University Hospital, Gwangju; ${ }^{2}$ Center for Aging and Geriatrics, Chonnam National University Medical School, Gwangju, Korea

\begin{abstract}
Objective To quantify changes in cardiopulmonary function using a lower body positive pressure supported (LBPPS) treadmill during the exercise tolerance test (ETT) in healthy subjects before applying the LBPPS treadmill in patients with gait problems.

Methods We evaluated 30 healthy subjects who were able to walk independently. The ETT was performed using the Modified Bruce Protocol (stages 1-5) at four levels (0\%, 40\%, 60\%, and $80 \%$ ) of LBPPS. The time interval at each level of the LBPPS treadmill test was 20 minutes to recover to baseline status. We measured systolic blood pressure, diastolic blood pressure, peak heart rate (PHR), rating of perceived exertion (RPE), metabolic equivalents (METs), and oxygen consumption rate $\left(\mathrm{VO}_{2}\right)$ during each LBPPS condition.

Results Systolic blood pressure increased as the LBPPS level was increased (40\% to $80 \%$ ). PHR, RPE, METs, and $\mathrm{VO}_{2}$ were negatively associated with the LBPPS condition, although they were not always significant different among the LBPPS levels. The equation from a random effect linear regression model was as follows: $\mathrm{VO}_{2}(\mathrm{~mL} / \mathrm{kg} /$ $\min )=(2.75 \times$ stage $)+(-0.14 \times$ LBPPS level $)+11.9\left(\mathrm{r}^{2}=0.69\right)$.

Conclusion Detection of the changes in physiological parameters during a submaximal ETT using the LBPPS system may be helpful for applying the LBPPS treadmill in patients who cannot perform the ETT due to gait problems, even at submaximal intensity.
\end{abstract}

Keywords Exercise test, Oxygen consumption, Metabolic equivalent, Rehabilitation, Gait

\section{INTRODUCTION}

The treadmill is used for the exercise tolerance test
(ETT) to objectively evaluate the physiological response of patients to cardiac rehabilitation [1]. However, many patients cannot complete the ETT due to general weak-

\footnotetext{
Received January 19, 2016; Accepted April 22, 2016

Corresponding author: Jae-Young Han

Department of Physical and Rehabilitation Medicine, Chonnam National University Medical School \& Hospital, 42 Jebong-ro, Dong-gu, Gwangju 61469, Korea. Tel: +82-62-220-5198, Fax: +82-62-228-5975, E-mail: rmhanjy@daum.net

ORCID: Ka-Young Lee (http://orcid.org/0000-0002-5521-5565); Jae-Young Han (http://orcid.org/0000-0003-1672-8875); Ji-Hyun Kim (http://orcid. org/0000-0003-4892-6753); Dong-Ju Kim (http://orcid.org/0000-0003-3695-3332); In-Sung Choi (http://orcid.org/0000-0003-3967-9201).
}

(c) This is an open-access article distributed under the terms of the Creative Commons Attribution Non-Commercial License (http://creativecommons.org/ licenses/by-nc/4.0) which permits unrestricted noncommercial use, distribution, and reproduction in any medium, provided the original work is properly cited. Copyright (c) 2016 by Korean Academy of Rehabilitation Medicine 
ness, motor weakness associated with previous disease (e.g., stroke, traumatic spinal cord injury, or Parkinson disease), or pain. Therefore, it is necessary to develop a novel device that enables patients with these disabilities to perform the ETT.

The body weight supported treadmill (BWST) has long been used in many rehabilitative areas. Early mobilization and training are required after lower extremity surgery, but patients cannot walk while they are bearing their body weight due to postoperative pain, instability, or motor weakness [2]. Gait training using the BWST is useful because the ground reaction force is reduced with normal ranges of motion of the knee and ankle joints [2]. Some studies have shown that the BWST improves gait performance in children with cerebral palsy, lumbar disc herniation, knee osteoarthritis, and in elderly people [36]. The BWST also improves gait outcome and clinical status in patients with Parkinson disease, stroke, and spinal cord injury [7-11].

The BWST system can also be used for the ETT to evaluate subjects with gait problems due to musculoskeletal disease or neurological problems. Traditionally, a harness system has been used to support body weight [12] The harness system consists of overhead suspension and chest corset with straps attached around the hips and thighs $[3,10]$. The patients feel uncomfortable when the overhead suspension lifts the body, particularly in the inguinal area, which may reduce training compliance $[3,13]$. Thus, the harness system has many limitations for its clinical application. A lower body positive pressure support (LBPPS) system has recently emerged as a system that can replace the harness system. This system has a waist-high inflatable chamber and a treadmill. The subject wears neoprene shorts that zip into the chamber, creating an airtight seal around the subject's waist. During training, positive pressure inflates the chamber, and the difference in pressure around the waist seal produces a lifting force [12]. The LBPPS makes the patient feel more comfortable and it allows for a kinematic walking pattern similar to overground walking. Patients with a lower heart rate can also train using the LBPPS than when using the harness system $[12,13]$. Thus, the LBPPS system could be a safe and effective way to train patients with disabilities. This system is thought to be useful for the ETT.

The LBPPS system may produce a different physiological response compared to that produced by other body weight support systems. The effect of the LBPPS is partly attributable to transport of blood to the heart, and hence, it increases cardiac preload and stroke volume because of the positive pressure created on the subject's lower extremities [14]. Therefore, an LBPPS treadmill might be harmful to patients with coronary artery disease.

It could be advantageous to apply the LBPPS device to the ETT in subjects with gait problems before beginning phase II cardiac rehabilitation. However, few studies have used the LBPPS system for cardiac rehabilitation. It is necessary to determine the physiological responses of cardiac patients to the LBPPS system before applying it clinically. A few studies have been conducted to evaluate the LBPPS treadmill test, but they had some limitations. Gojanovic et al. [15] reported physiological changes during maximal treadmill exercise intensities using LBPPS. However, in order to apply LBPPS during the ETT before phase II cardiac rehabilitation, the physiological changes that occur at submaximal exercise intensity should be determined. Hoffman and Donaghe [16] determined the equations that define $\mathrm{VO}_{2}$ using only speed and LBPPS level as variants, without considering the inclination which changes as the stage of the Modified Bruce Protocol increases.

The aim of this study was to quantify changes in cardiopulmonary function using the LBPPS treadmill during the submaximal ETT in healthy subjects in order to identify clinically meaningful changes and predict adverse events.

\section{MATERIALS AND METHODS}

\section{Subjects}

Thirty healthy subjects (13 men and 17 women; age, 23-73 years) who were able to walk independently participated in this study (Table 1). Eight subjects were on antihypertensive medication with stable blood pressure (Table 1). The exclusion criteria included known cardiac or pulmonary disease, musculoskeletal disorder, or an acute disease preventing test participation.

This study was approved by the Institutional Review Board of Chonnam National University Hospital (CNUH2014-159) and it was conducted according to the Declaration of Helsinki. All subjects signed the informed consent form. 
Table 1. Characteristics of the selected subjects

\begin{tabular}{|cc|}
\hline Characteristic & Value \\
\hline Age (yr) & $56.57 \pm 14.43$ \\
\hline 55 & $20(66.7)$ \\
\hline Sex (male) & $13(43.3)$ \\
\hline HTN & $8(26.7)$ \\
\hline DM & $4(13.3)$ \\
\hline Height $(\mathrm{cm})$ & $163.1 \pm 9.26$ \\
\hline Weight $(\mathrm{kg})$ & $63.65 \pm 11.75$ \\
\hline MMT & All in 5 grade \\
\hline ROM & All in full range \\
\hline 10MWT_CS (s) & $7.77 \pm 1.16$ \\
\hline 10MWT_MS (s) & $5.92 \pm 0.93$ \\
\hline
\end{tabular}

Values are expressed as mean \pm standard deviation or number (\%).

HTN, hypertension; DM, diabetes mellitus; MMT, manual muscle test; ROM, range of motion; 10MWT_CS, 10-m walking test at comfortable speed; 10MWT_MS, 10-m walking test at maximal speed.

\section{Experimental procedure}

The subjects visited our clinic and their cardiopulmonary function was evaluated. Before the ETT, the subjects were assessed for muscle strength via manual muscle testing and major joint range of motion, which were in the normal range (Table 1).

A 10-m walking test was conducted at comfortable and maximal speed to evaluate the walking ability. The protocol started at least 2 hours after a meal and each subject completed 10 minutes of warm-up and cool-down exercises.

An incremental submaximal exercise tolerance test was performed on the AlterG M320 (AlterG Inc., Fremont, CA, USA) at four different LBPPS levels (LBPPS $0 \%$, LBPPS $40 \%$, LBPPS $60 \%$, and LBPPS $80 \%$ ) in a randomized order (Fig. 1). LBPPS $0 \%$ is the conventional treadmill under the impact of no antigravity, LBPPS $40 \%$ is the treadmill in which the positive pressure on the lower body lifts $40 \%$ of the body weight, LBPPS $60 \%$ is the treadmill that lifts $60 \%$ of the body weight, and LBPPS $80 \%$ is the treadmill that raises $80 \%$ of the body weight using antigravity. The exercise test was performed using the Modified Bruce Protocol (stages 1-5). Each stage lasted 3 minutes. Stage 1 progressed at a velocity of $1.7 \mathrm{mph}$ and $0 \%$ grade. Stages 2 and 3 increased the grade to $5 \%$ and $10 \%$ without changing the velocity. Then, the velocity was increased by

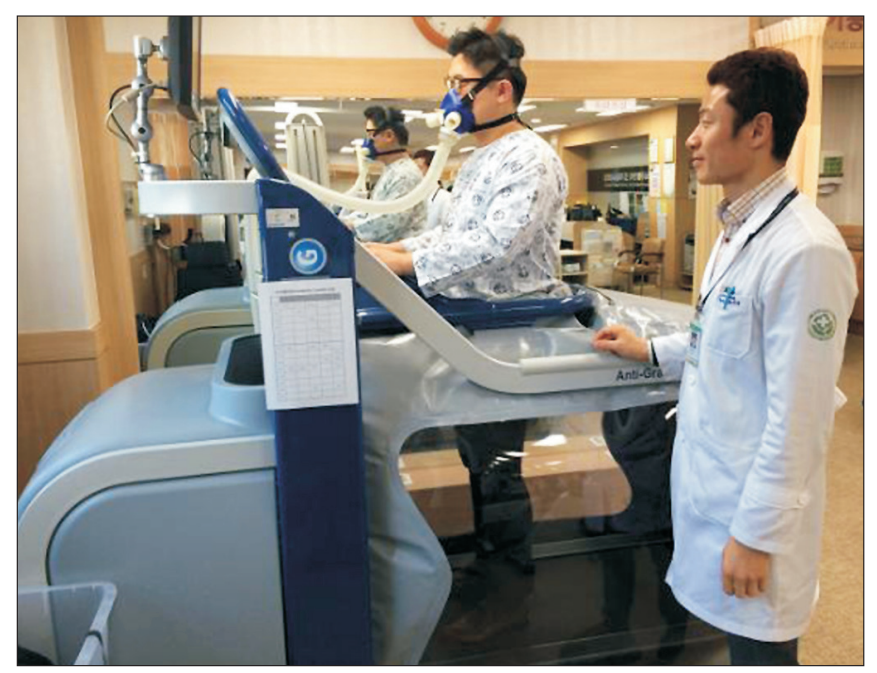

Fig. 1. A subject performing the exercise tolerance test on the lower body positive pressure supported treadmill AlterG M320 (AlterG Inc., Fremont, CA, USA).

Table 2. Modified Bruce Protocol

\begin{tabular}{cccc}
\hline Stage & Speed (mph) & Grade (\%) & Duration (min) \\
\hline 1 & 1.7 & 0 & 3 \\
2 & 1.7 & 5 & 3 \\
3 & 1.7 & 10 & 3 \\
4 & 2.5 & 12 & 3 \\
5 & 3.4 & 14 & 3 \\
\hline
\end{tabular}

$0.8-0.9 \mathrm{mph}$ and the grade was increased by $2 \%$ every 3 minutes. The subjects were instructed to complete stage 5 of the test (Table 2).

A 20-minute time period to allow the subject to recover to baseline status was provided after each level of the LBPPS treadmill test. We measured systolic and diastolic blood pressures (SBP and DBP) during the last minute of the stage with an automatic device. Metabolic equivalents (METs), heart rate, and oxygen consumption rate $\left(\mathrm{VO}_{2}\right)$ were measured continuously by the TrueOne 2400 metabolic system (Parvo Medics, Sandy, UT, USA) during the entire test (Fig. 2). Subjects provided the Borg rating of perceived exertion (RPE) during the last 30 seconds of each stage. The test was stopped when the subject complained of dizziness, headache, or chest pain. Four subjects could not complete the test due to leg pain. All testing was supervised by an experienced ETT trainer. 


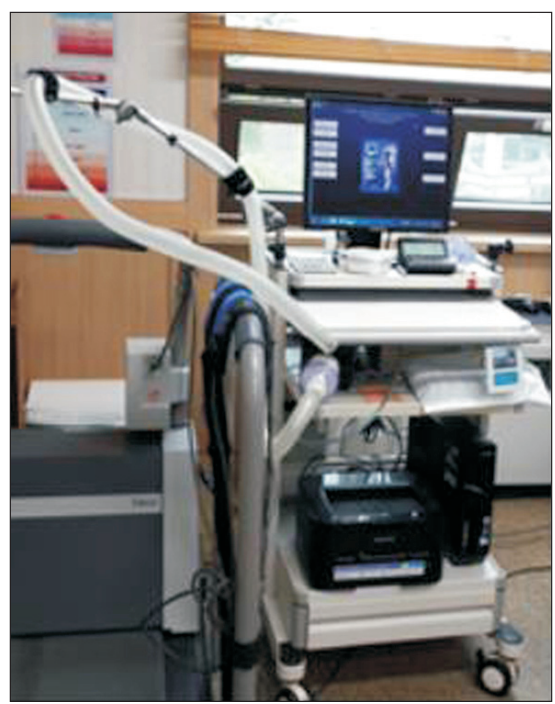

Fig. 2. The TrueOne 2400 metabolic system (Parvo Medics, Sandy, UT, USA), which measures the cardiometabolic parameters.

\section{Statistical analysis}

One-way analysis of variance and the least significant difference test were performed to compare SBP and DBP, peak heart rate (PHR), RPE, METs, and $\mathrm{VO}_{2}$. The p-values $<0.05$ were considered statistically significant. All statistical analyses were performed using SPSS ver. 23 software (IBM Corp., Armonk, NY, USA). A random effects linear regression analysis was performed to predict an equation that describes $\mathrm{VO}_{2}$ with the stage and body weight supporting level as variables using STATA ver. 12.1 software (Stata Corp., College Station, TX, USA).

\section{RESULTS}

Twenty-six out of the total 30 subjects completed the LBPPS treadmill test. Three subjects stopped the test at stage 4 of LBPPS $0 \%$, and the remaining one subject dropped out at stage 3 of LBPPS $0 \%$. They could not complete the test due to leg pain.

SBP at LBPPS $0 \%$ was significantly higher than that at LBPPS $40 \%$ and $60 \%$ during stages 1 and $2(\mathrm{p}=0.001)$. SBPs measured during the LBPPS $40 \%, 60 \%$, and $80 \%$ were significantly different during stages $3-5$ from those measured at LBPPS $0 \%(\mathrm{p}<0.008)$. Except for LBPPS $0 \%$, as the LBPPS level increased in other words, the degree of lifting of the body weight became greater, SBP showed an increasing tendency. However, SBP displayed no sig-

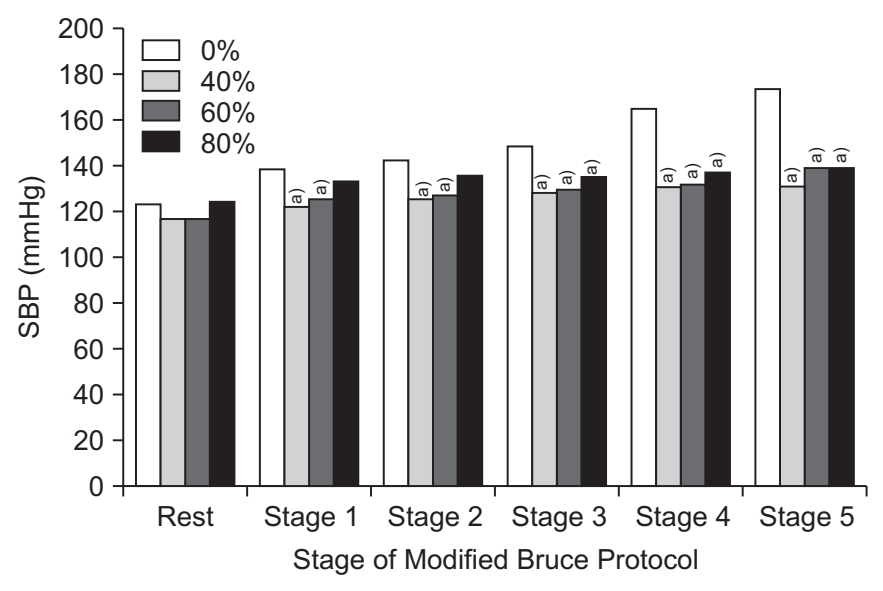

Fig. 3. Systolic blood pressure (SBP) was significantly lower in the lower body positive pressure support (LBPPS) state than that on the regular treadmill at every stage $\left({ }^{a)} p<0.05\right.$ compared to LBPPS $0 \%$ ).

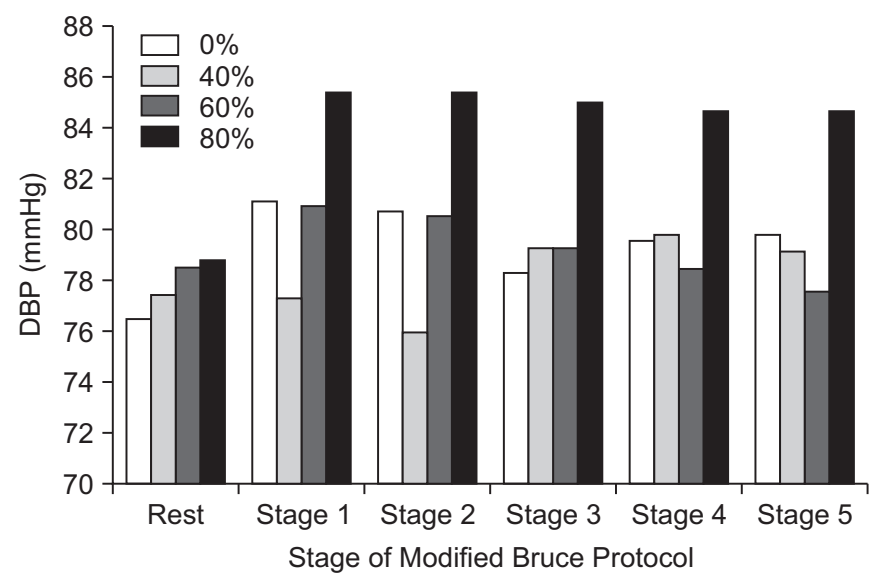

Fig. 4. Diastolic blood pressure (DBP) was not different among the body weight support conditions at any stage $(\mathrm{p}>0.05)$.

nificant association with the level of lower body weight support from LBPPS $40 \%$ to LBPPS $80 \%$ at any stage (Fig. $3)$.

DBP was not different among the body weight support conditions at any stage ( $p>0.05$ ) (Fig. 4).

PHR at LBPPS $0 \%$ was significantly higher than that at the other levels through stage $5(\mathrm{p}<0.008)$. A higher LBPPS level during stages 4 and 5 resulted in a lower PHR under all LBPPS conditions. Nevertheless, no statistical difference was detected within the LBPPS levels ( $p>0.05$ ) (Fig. 5).

RPE at LBPPS $0 \%$ was significantly higher than that at the other three LBPPS conditions during stages 2 and 3. RPE at 


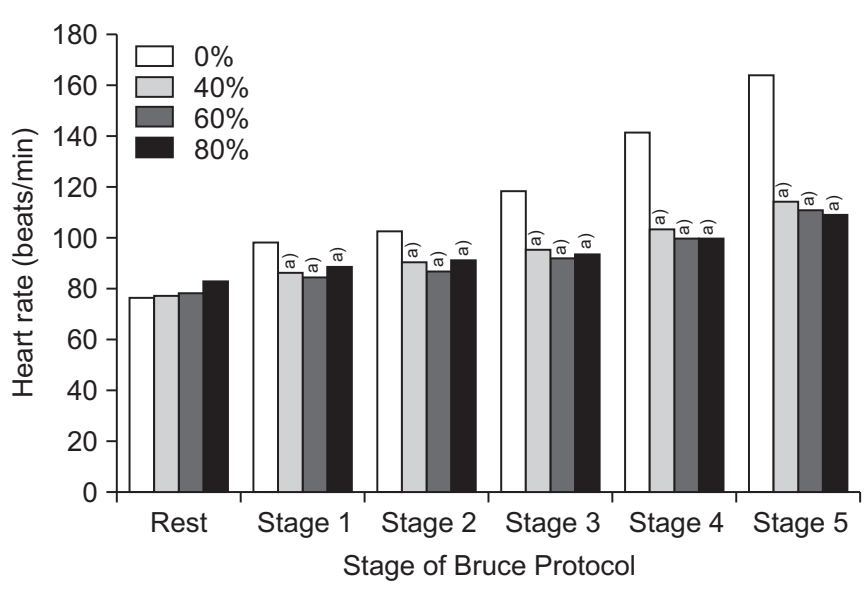

Fig. 5. Peak heart rate (PHR) on the general treadmill was significantly higher than that during the other conditions until completion of stage $5\left({ }^{a} \mathrm{p}<0.05\right.$ compared to lower body positive pressure support [LBPPS] 0\%). PHR decreased at higher LBPPS levels during stages 4 and 5, although no statistically differences were detected within the LBPPS levels.

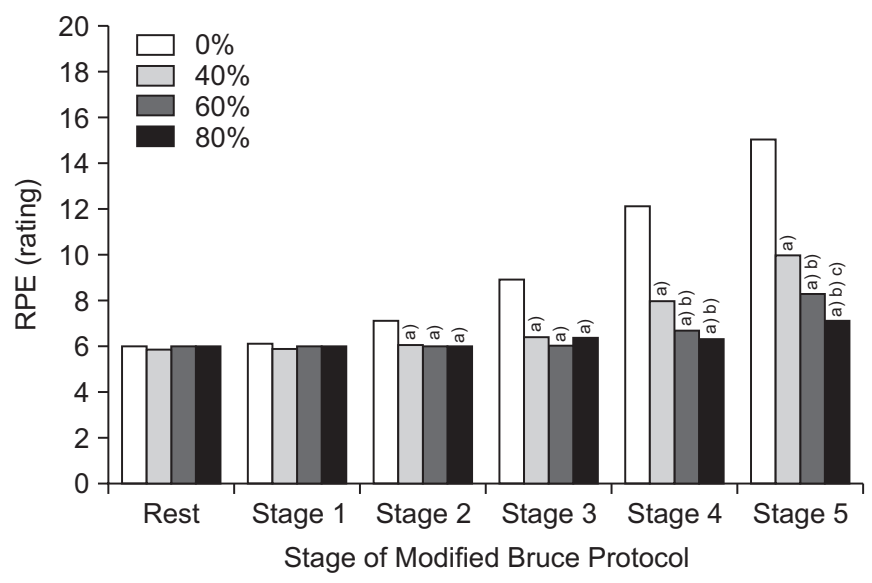

Fig. 6. Rating of perceived exertion (RPE) on the general treadmill was significantly higher than that during the other three LBPPS conditions during stages 2 and 3. RPE on the general treadmill and at LBPPS $40 \%$ during stage 4 was significantly different from that at LBPPS $60 \%$ and $80 \%$. RPE decreased significantly as the LBPPS level was increased $\left({ }^{a)} \mathrm{p}<0.05\right.$ compared to LBPPS $0 \%,{ }^{\text {b) }} \mathrm{p}<0.05$ compared to LBPPS $40 \%,{ }^{c} \mathrm{p}<0.05$ compared to LBPPS $60 \%)$. LBPPS, lower body positive pressure support.

LBPPS $0 \%$ and LBPPS $40 \%$ during stage 4 was significantly different from that at LBPPS $60 \%$ and $80 \%(p<0.002)$. As the LBPPS level increased, the RPE decreased and the level of support showed a close association during stage 5 (p<0.02) (Fig. 6).

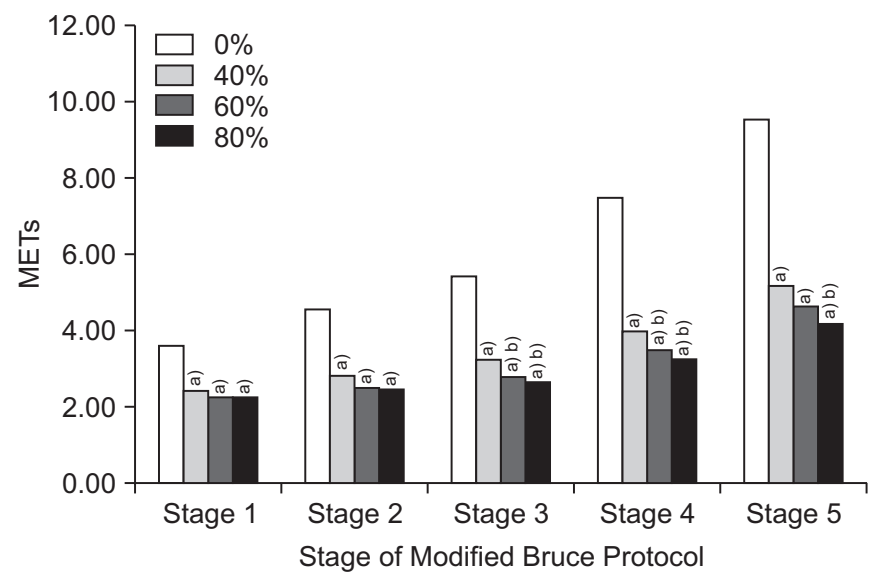

Fig. 7. Metabolic equivalents (METs) on the general treadmill were significantly higher than those at other LBPPS levels during stages 1 and 2. METs at LBPPS $0 \%$ and $40 \%$ during stages 3 and 4 were significantly different from those at LBPPS $60 \%$ and $80 \%$. The general treadmill showed significantly higher METs than those at the other LBPPS levels during stage 5 . Significant differences were detected between METs at LBPPS $40 \%$ and $80 \%$, but not between LBPPS $40 \%$ and $60 \%\left({ }^{\text {a) }} \mathrm{p}<0.05\right.$ compared to LBPPS $0 \%,{ }^{b)} \mathrm{p}<0.05$ compared to LBPPS $40 \%$ ). LBPPS, lower body positive pressure support.

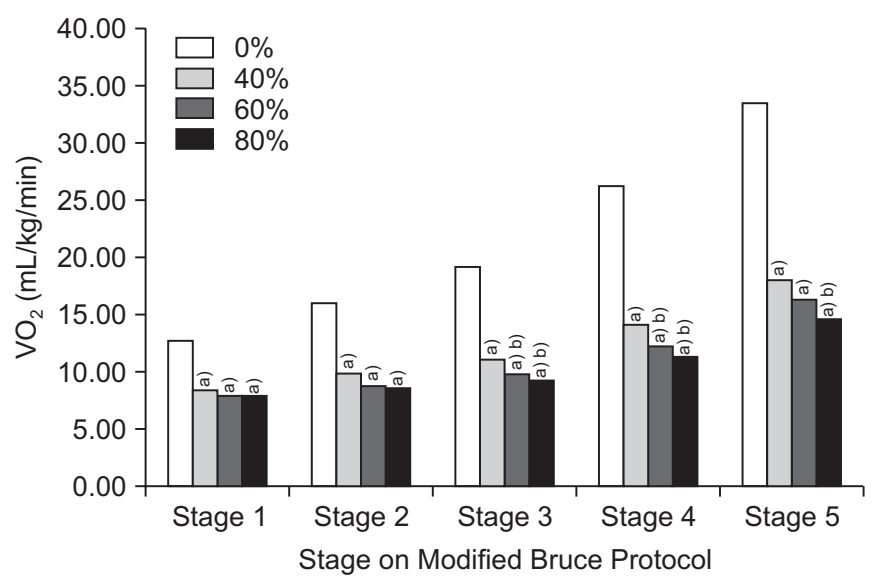

Fig. 8. Oxygen consumption rate $\left(\mathrm{VO}_{2}\right)$ on the general treadmill was significantly higher than that during other LBPPS treadmill conditions at every stage. $\mathrm{VO}_{2}$ at LBPPS $40 \%$ was also significantly higher than that at LBPPS $60 \%$ and $80 \%$ during stages 3 and $4 . \mathrm{VO}_{2}$ at LBPPS $40 \%$ was significantly higher during stage 5 than that at LBPPS $80 \%$, but not compared to that at LBPPS $60 \%\left({ }^{a}\right) \mathrm{p}<0.05$ compared to LBPPS $0 \%$, ${ }^{b)} \mathrm{p}<0.05$ compared to LBPPS $40 \%$ ). LBPPS, lower body positive pressure support.

Lower body weight support showed a decreasing METs and $\mathrm{VO}_{2}$ trend as the LBPPS level was increased. METs 
and $\mathrm{VO}_{2}$ at LBPPS $0 \%$ were significantly higher during stages 1 and 2 than those at other lower body supported treadmill conditions $(\mathrm{p}<0.001)$. METs and $\mathrm{VO}_{2}$ at LBPPS $0 \%$ and LBPPS $40 \%$ were significantly higher than those at LBPPS $60 \%$ and $80 \%$ during stages 3 and $4(\mathrm{p}<0.03)$. METs and $\mathrm{VO}_{2}$ at LBPPS $0 \%$ were significantly different from those at the other three LBPPS levels during stage 5 $(\mathrm{p}<0.001)$. Also, METs and $\mathrm{VO}_{2}$ at LBPPS $40 \%$ were significantly higher than those at LBPPS $80 \%(\mathrm{p}=0.003)$, but not compared to those at LBPPS $60 \%$ during stage 5 (Figs. 7, 8).

The equation from the random effects linear regression model that describes $\mathrm{VO}_{2}(\mathrm{~mL} / \mathrm{kg} / \mathrm{min})$ as a function of the Modified Bruce Protocol stage and the level of body weight support was determined based on data from the 26 subjects who completed the tests.

$$
\begin{aligned}
\mathrm{VO}_{2}(\mathrm{~mL} / \mathrm{kg} / \mathrm{min})= & (2.75 \times \text { stage })+(-0.14 \times \text { LBPPS level })+ \\
& 11.9\left(\mathrm{r}^{2}=0.69\right)
\end{aligned}
$$

\section{DISCUSSION}

Our study was designed to determine the changes in physiological responses during the LBPPS treadmill test in healthy subjects and to identify correlations between different body weight support levels on the treadmill test using the Modified Bruce Protocol for planning a cardiac rehabilitation program. To the best of our knowledge, this is the first study to use the Modified Bruce Protocol for the ETT under the LBPPS condition.

In this study, SBP was significantly lower in the LBPPS state than that at LBPPS $0 \%$. However, exposure to higher LBPPS levels increased the SBP, possibly due to translocation of blood volume from the lower extremities to the chest for increased cardiac preload and stroke volume [14]. LBPPS also raises the intramuscular pressure, which activates the muscle mechanoreflex, resulting in increased BP [17]. Furthermore, positive pressure on the lower extremities limits the blood supply to the active muscles, and hence metabolites accumulate and a pressure metaboreflex is triggered $[18,19]$.

Previous studies showed that the application of BWST reduces PHR. Nishiyasu et al. [20] reported that heart rate in the upright position drops during LBPPS, but no change was observed in the supine position. Heart rate may drop due to decreased gait energy cost as a result of body weight unloading [21]. The baroreceptor reflex in response to transport of blood volume from the lower extremities to the chest during LBPPS can decrease the heart rate [14]. In our study, PHR decreased at higher LBPPS levels, although no statistical difference was observed within LBPPS levels.

Body weight support caused a significant drop in $\mathrm{VO}_{2}$ values, and $\mathrm{VO}_{2}$ tended to decrease as the degree of LBPPS increased. This finding is similar to that in other studies, although a significant difference in $\mathrm{VO}_{2}$ between each LBPPS condition until stage 5 was not always observed $[13,22,23] . \mathrm{VO}_{2}$ consistently increased at every LBPPS level until the subject completed the test. McNeill et al. [22] found that $\mathrm{VO}_{2}$ decreases significantly with increasing LBPPS level and it increases with increasing velocity. In addition, the drop in $\mathrm{VO}_{2}$ was attenuated as the LBPPS level was increased. We suggest that a higher level of LBPPS does not affect the cardiopulmonary demand during the ETT with body weight support on a treadmill.

RPE decreased significantly during stage 5 as the LBPPS level was increased. Ruckstuhl et al. [23] reported that $\mathrm{VO}_{2}$, heart rate, and RPE decrease during LBPPS, and greater drops were detected at higher exercise speeds, which made training to the maximal intensity easier. As shown in our study, METs and RPE were much lower under the LBPPS condition than those on the regular treadmill test. Therefore, it may be difficult to achieve the patient's symptom-limited state with LBPPS. We suggest that it is appropriate to conduct a submaximal ETT with the LBPPS system in patients with a gait disturbance before the early phase of cardiac rehabilitation.

In this study, we calculated an equation using random effects linear regression to estimate $\mathrm{VO}_{2}$ using the stage and LBPPS level as variates and it was useful for an actual exercise treadmill test. Obtaining $\mathrm{VO}_{2}$ by inserting the stage and LBPPS level in the equation could help the physicians to determine METs, so that they can prescribe exercise intensity in cardiac rehabilitation. This is significant because previous equations did not use the protocol stage as a variant, which includes inclination. The coefficient of determination $\left(r^{2}\right)$ is closer to 1 as the correlation within variates becomes stronger. In our study, the $r^{2}$ was 0.69 , which is higher than 0.65 but not very close to 1 . This result may be due to broad variations in age, physical condition, or medications used by our subjects. Therefore, a future study with more homogeneous sub- 
jects should be performed to determine an equation with a higher correlation within variates.

Hautala et al. [24] created an equation for METs at maximal exercise capacity using submaximal exercise results. Also, we could prescribe exercise intensity in patients undergoing cardiac rehabilitation by determining $\mathrm{VO}_{2}$ at maximal intensity using the equation proposed by Hautala et al.

Our study demonstrates that the LBPPS system allows patients to have a less stressful ETT and to maintain cardiorespiratory demand.

The ETT is used to identify the METs that a patient can achieve during cardiac rehabilitation [1]. However, many limitations prevent patients from completing the ETT. Patients with walking problems due to stroke, spinal cord injury, neuromuscular disease, or surgery on the lower extremity cannot participate in the ETT. Some devices have been employed to support body weight during walking, including harness suspension systems and water immersion $[2,13]$. A harness system consists of overhead suspension and a chest corset with straps attached around the hips and thighs, and it is used frequently because it can apply a purely vertical force to the subject $[3,10]$. However, it has not been used during extended training because it can cause discomfort in the inguinal area and can interrupt circulation [13]. Water immersion is useful during rehabilitation; however, the drag forces during water training act in the opposite direction to the movement and seriously alter gait velocity, timing, joint kinetics, joint kinematics, and muscle activity [13]. Water immersion may increase the risk of infection during immediate postoperative rehabilitation in patients who had surgery on the lower extremity [2].

The LBPPS system, which has a waist-high inflatable chamber and treadmill, has replaced previous body weight support devices. The patient wears neoprene shorts that zip into a chamber, creating an airtight seal around the patient's waist, and positive pressure is used to inflate the chamber and produce a lifting force [12]. The LBPPS system can be used during the ETT at submaximal intensity in patients with a gait disturbance or general weakness before beginning the early phase of cardiac rehabilitation.

This study had several limitations. First, the sample size was small. A more detailed and homogeneous group of subjects would help increase the correlation between variates. We could not evaluate the maximal physiological response during stage 6 , although the purpose was to evaluate the submaximal response. This was due to structural limitations of the LBPPS machines available in the market in which the inclination cannot be increased; hence, new machinery is necessary for applying the ETT under maximal intensity.

Further research should be designed to define $\mathrm{VO}_{2}$ using speed and inclination as variates. An evaluation of maximal intensity ETT is also necessary based on our results.

In this study, it was difficult to obtain PHR as the LBPPS level increased because ETT was performed in healthy subjects who did not have gait problems. However, it may be possible to evaluate PHR at lower inclination and slower velocity in patients with gait problems. Therefore, it is necessary to define physiologic responses to ETT with LBPPS in patients of different ages and with different types of gait difficulties.

In conclusion, evaluation of the changes in physiological parameters during submaximal ETT using the LBPPS system may be helpful for applying the LBPPS treadmill in patients who cannot perform the ETT due to gait problems and for prescribing exercise intensity or type in patients. Furthermore, cardiac rehabilitation could be applied more extensively with ease and safety using the LBPPS treadmill.

\section{CONFLICT OF INTEREST}

No potential conflict of interest relevant to this article was reported.

\section{ACKNOWLEDGMENTS}

This study was supported by a grant (No. CRI-110561) by Chonnam National University Hospital Research Institute of Clinical Medicine. This study was financially supported by the Electronic Medical Equipment Part \& Material Industrialization Foundation Construction Program through the Ministry of Trade, Industry, \& Energy (MOTIE) and the Korea Institute for Advancement of Technology (KIAT) (No. R0002662). 


\section{REFERENCES}

1. Dunagan J, Adams J, Cheng D, Barton S, Bigej-Cerqua J, Mims L, et al. Development and evaluation of a treadmill-based exercise tolerance test in cardiac rehabilitation. Proc (Bayl Univ Med Cent) 2013;26:24751.

2. Cutuk A, Groppo ER, Quigley EJ, White KW, Pedowitz RA, Hargens AR. Ambulation in simulated fractional gravity using lower body positive pressure: cardiovascular safety and gait analyses. J Appl Physiol (1985) 2006;101:771-7.

3. Kurz MJ, Corr B, Stuberg W, Volkman KG, Smith N. Evaluation of lower body positive pressure supported treadmill training for children with cerebral palsy. Pediatr Phys Ther 2011;23:232-9.

4. Takacs J, Anderson JE, Leiter JR, MacDonald PB, Peeler JD. Lower body positive pressure: an emerging technology in the battle against knee osteoarthritis? Clin Interv Aging 2013;8:983-91.

5. Moore MN, Vandenakker-Albanese C, Hoffman MD. Use of partial body-weight support for aggressive return to running after lumbar disk herniation: a case report. Arch Phys Med Rehabil 2010;91:803-5.

6. Mangione KK, Axen K, Haas F. Mechanical unweighting effects on treadmill exercise and pain in elderly people with osteoarthritis of the knee. Phys Ther 1996;76:387-94.

7. Ganesan M, Sathyaprabha TN, Pal PK, Gupta A. Partial body weight-supported treadmill training in patients with Parkinson disease: impact on gait and clinical manifestation. Arch Phys Med Rehabil 2015;96:1557-65.

8. Rose MH, Lokkegaard A, Sonne-Holm S, Jensen BR. Improved clinical status, quality of life, and walking capacity in Parkinson's disease after body weight-supported high-intensity locomotor training. Arch Phys Med Rehabil 2013;94:687-92.

9. Sousa CO, Barela JA, Prado-Medeiros CL, Salvini TF, Barela AM. Gait training with partial body weight support during overground walking for individuals with chronic stroke: a pilot study. J Neuroeng Rehabil 2011;8:48.

10. Visintin M, Barbeau H, Korner-Bitensky N, Mayo NE. A new approach to retrain gait in stroke patients through body weight support and treadmill stimula- tion. Stroke 1998;29:1122-8.

11. Senthilvelkumar T, Magimairaj H, Fletcher J, Tharion G, George J. Comparison of body weight-supported treadmill training versus body weight-supported overground training in people with incomplete tetraplegia: a pilot randomized trial. Clin Rehabil 2015;29:429.

12. Ruckstuhl H, Kho J, Weed M, Wilkinson MW, Hargens AR. Comparing two devices of suspended treadmill walking by varying body unloading and Froude number. Gait Posture 2009;30:446-51.

13. Grabowski AM. Metabolic and biomechanical effects of velocity and weight support using a lower-body positive pressure device during walking. Arch Phys Med Rehabil 2010;91:951-7.

14. Sota T, Matsuo S, Uchida Y, Hagino H, Kawai Y. Effects of lower body positive pressure on cardiovascular responses during walking in elderly women. Physiol Res 2013;62:653-62.

15. Gojanovic B, Cutti P, Shultz R, Matheson GO. Maximal physiological parameters during partial bodyweight support treadmill testing. Med Sci Sports Exerc 2012;44:1935-41.

16. Hoffman MD, Donaghe HE. Physiological responses to body weight: supported treadmill exercise in healthy adults. Arch Phys Med Rehabil 2011;92:960-6.

17. Fu Q, Sugiyama Y, Kamiya A, Shamsuzzaman AS, Mano T. Responses of muscle sympathetic nerve activity to lower body positive pressure. Am J Physiol 1998;275(4 Pt 2):H1254-9.

18. Macias BR, D'Lima DD, Cutuk A, Patil S, Steklov N, Neuschwander TB, et al. Leg intramuscular pressures and in vivo knee forces during lower body positive and negative pressure treadmill exercise. J Appl Physiol (1985) 2012;113:31-8.

19. Williamson JW, Mitchell JH, Olesen HL, Raven PB, Secher NH. Reflex increase in blood pressure induced by leg compression in man. J Physiol 1994;475:351-7.

20. Nishiyasu T, Nagashima K, Nadel ER, Mack GW. Effects of posture on cardiovascular responses to lower body positive pressure at rest and during dynamic exercise. J Appl Physiol (1985) 1998;85:160-7.

21. Grabowski A, Farley CT, Kram R. Independent metabolic costs of supporting body weight and accelerating body mass during walking. J Appl Physiol (1985) 2005;98:579-83. 
22. McNeill DK, Kline JR, de Heer HD, Coast JR. Oxygen consumption of elite distance runners on an antigravity treadmill. J Sports Sci Med 2015;14:333-9.

23. Ruckstuhl H, Schlabs T, Rosales-Velderrain A, Hargens AR. Oxygen consumption during walking and running under fractional weight bearing conditions.
Aviat Space Environ Med 2010;81:550-4.

24. Hautala AJ, Kiviniemi AM, Karjalainen JJ, Piira OP, Lepojarvi S, Makikallio T, et al. Peak exercise capacity prediction from a submaximal exercise test in coronary artery disease patients. Front Physiol 2013;4:243. 